\title{
Intergenerational neurocognitive effects of intrauterine hyperglycemia on male offspring
}

\section{Kexin Zou}

Shanghai Jiao Tong University

Jun Ren

Zhejiang University

Junyu Zhang

Shanghai Jiao Tong University

Chengliang Zhou

Shanghai Jiao Tong University

Sisi Luo

Shanghai Jiao Tong University

\section{Chengxi Tan}

Shanghai Jiao Tong University

\section{Pingping Lv}

Zhejiang University

\section{Xiao Sun}

Shanghai Jiao Tong University

Jianzhong Sheng

Zhejiang University

Hefeng Huang ( $\square$ huanghefg@sjtu.edu.cn )

Shanghai Jiao Tong University

Xinmei Liu ( $\square$ nanlilac@hotmail.com )

Shanghai Jiao Tong University

Guolian Ding ( $\nabla$ dingguolian@hotmail.com )

Shanghai Jiao Tong University https://orcid.org/0000-0002-0099-9980

\section{Research}

Keywords: Cognition, DNA methylation, Epigenetic inheritance, Gestational diabetes mellitus, Hippocampus, Offspring

Posted Date: August 4th, 2020

DOI: https://doi.org/10.21203/rs.3.rs-49569/v1 
License: (c) (i) This work is licensed under a Creative Commons Attribution 4.0 International License. Read Full License 


\section{Abstract \\ Background}

Studies on human and animals suggest associations between gestational diabetes mellitus (GDM) with increased susceptibility to develop neurological disorders in offspring. However, researches have focused on the neurodevelopment consequences of the first filial (F1) offspring. We hypothesize that the intrauterine hyperglycemia exposure will alter epigenetic reprogramming in F1 sperm, and carry risks of passing on molecular defects to the second filial (F2).

\section{Results}

We found that intrauterine hyperglycemia exposure resulted in memory impairment in both F1 and F2 males from the F1-GDM male offspring. Transcriptome profiling of F1 and F2 hippocampi revealed that differentially expressed genes were enriched in learning, memory, cognition, neurotransmission, synaptic plasticity, and postsynaptic specialization. Again, enrichment curves computed by Gene set enrichment analysis (GSEA) of F1 hippocampi were highly consistent with F2. Interestingly, combined analysis of F1 sperm methylome and gene expression of F2 hippocampi screened out several hypermethylation-low expression genes which are associated with abnormal central nervous system development (particularly synapse development and homeostasis), such as Camk2b, Dlgap1, Wnt5a, Tubb2b and so on.

\section{Conclusions}

These findings implicate that the male germ line is a major player in transgenerational phenotypic transmission. Taken together, our results for the first time suggest that intrauterine hyperglycemia leads to transgenerational cognitive impairment, and, sperm methylome is a potential epigenetic mechanism for the effects of GDM.

\section{Background}

Accumulating evidence has suggested that the adverse developmental environment can increase the risk of chronic diseases in later life. Long-term postnatal health may be affected by metabolic experience in utero $[1,2]$. Intrauterine hyperglycemia is a major characteristic of gestational diabetes mellitus (GDM) and is associated with a high risk of diabetes in offspring [3]. In our previous study, we found that impaired glucose tolerance (IGT) in male offspring was more obvious than females, with parental characteristics and sex-specific transmission[4]. Our further study showed that the aberrant DNA methylation reprogramming occurs as early as day 13.5 in primordial germ cells (PGCs) of the first filial generation (F1), suggesting that intrauterine exposure alone is sufficient to cause the epigenetic changes in the second filial generation (F2) [5]. 
In humans, it is noteworthy that in addition to metabolic dysfunction, studies have documented that children of mothers with diabetes during pregnancy is associated with impaired cognitive ability [6-9], although one study failed to detect such association in another population [10]. It is also controversial whether the association between maternal diabetes in pregnancy and offspring cognitive outcomes can be fully explained by shared familial environmental factors or by an intrauterine biological mechanism [11].

Experimental investigations in animals indicated that uncontrolled diabetes mellitus was associated with morphological and functional alterations in the brain [12-14]. Hippocampus, a structure critical to cognitive processes, has been shown to undergo apoptotic cell death when subjected to hyperglycemic insult [15-17]. Diabetes during pregnancy strongly influences the regulation of both insulin-like growth factor-1 receptor (IGF-1R) and insulin receptor (InsR) in the rat hippocampus [15]. Maternal diabetes mellitus can also reduce expression of synaptophysin (SYP) in the developing hippocampus and cerebellar cortex of neonatal rats $[18,19]$. However, the intergenerational effect on the F2 offspring and the underlying molecular mechanism are unclear.

Epigenetic alterations regulate tissue-specific gene expression during growth and development without alterating the DNA sequence. DNA methylation primarily occurs on $\mathrm{CpG}$ dinucleotides and is generally associated with gene repression when positioned on the promoter region $[2,20]$. Our previous research has demonstrated that intrauterine hyperglycemia changed DNA methylation levels on the imprinted gene Igf2/H19 in F1 pancreatic islet, which was further transmitted to F2 through F1 germ cells [4]. Therefore, we hypothesize that the hyperglycemic intrauterine environment of GDM could result in a high risk of cognitive impairment in F2 offspring by affecting the development or function of hippocampus through altering DNA methylation in F1 germ cells. We focused on the male offspring in this study because our previous GDM mouse model of intrauterine hyperglycemia indicates that male offspring was more susceptible to such intergenerational effects than females.

\section{Materials And Methods}

\section{Mice}

All animal protocols were reviewed and approved by the Zhejiang University Animal Care and Use Committee. At the age of 8 weeks, virgin female ICR mice $(n=60)$ were mated with normal males. Onset of pregnancy was determined by the presence of a copulation plug after overnight mating (designated as day 0 [D0] of pregnancy). After a 12-h fast, the females were randomly divided into a control group and an intrauterine hyperglycemia group with GDM (GDM group). Mice in the GDM group were injected with a single intraperitoneal injection of streptozotocin (STZ; Sigma, St. Louis, MO) in $0.1 \mathrm{mmol} / \mathrm{L}$ citrate buffer $(\mathrm{pH} \mathrm{4.5)}$ at a dose of $150 \mathrm{mg} / \mathrm{kg}$ body wt. Control pregnant females received an equal volume of citrate buffer. Diabetes was confirmed by measurement of blood glucose concentration via the tail vein as previously described [4]. The pregnant mice were allowed to deliver spontaneously. The litter size was randomly reduced to 10 at birth to assure uniformity. The pups from the GDM group were fostered by 
normoglycemic females until they were weaned at the age of 3 weeks. The F1 adults of control (F1-C) female and F1-C male were intercrossed to obtain F2 offspring of control (F2-C) group. The F1-C female and F1 adults of GDM (F1-GDM) male mice were intercrossed to obtain F2 offspring of GDM (F2-GDM) group.

\section{Behavioral tests}

All the behavior tests were done from 3-month-old during 12pm-6pm in a dim environment, unless specifically mentioned. Mice were given one-week interval to recover from the first behavioral test to have a next behavioral test. (1) The Open Field (OF) test consisted of a clear dark-colored polyvinyl plastic-wall arena measuring $50 \times 50 \mathrm{~cm}$ with $40 \mathrm{~cm}$ high walls and a dark floor. The center of $20 \times 20 \mathrm{~cm}$ was demarcated on a computer, leaving a surrounding outer zone of $30 \mathrm{~cm}$ width. The behavior of each mouse was recorded with Noldus tracking software. (2) The Y-maze tested spontaneous spatial recognition as a hippocampus-dependent memory test. The Y-maze, a horizontal maze consisting of three arms $(40 \mathrm{~cm} \times 3 \mathrm{~cm} \times 12 \mathrm{~cm})$, has arms symmetrically disposed at a $120^{\circ}$ angle. The floor and walls of the maze were made with a dark-colored opaque polyvinyl plastic. Mice were placed in one arm. The sequence (e.g., $A B C A B$ ) and number of arm entry were manually recorded for each mouse for an 8min period. Entry into all three arms on consecutive choices was defined as an actual alteration (i.e., $A B C$, $\mathrm{CAB}$, or $\mathrm{BCA}$, but not $\mathrm{BAB})$. Between tests, maze arms were cleaned to remove residual odors. The alternation percentage was defined as the following equation: $\%$ alternation $=[$ (number of alternations) $/$ (total arm entries -2 ) $] \times 100$. Percentage arm alteration is an established behavioral assay

for short-term spatial memory. (3) In Novel Subject of Recognition (NOR) test, a mouse is presented with two similar objects in the open field during the familiarization session for $8 \mathrm{~min}$, and then one of the two objects was replaced by a novel object during a second session in which mouse can behave freely for $8 \mathrm{~min}$. NOR discrimination index (DI) was calculated by the following formula: (Time exploring novel object - Time exploring familiar object) / Total object exploration time. The amount of time taken to explore the new object provides an index of recognition memory. Higher DI indicates better recognition memory. (4) The Object-in-place task (OiPT) was comprised of an acquisition phase and a test phase separated by a 30 min delay. In the acquisition phase, the subjects were presented with four different objects (A-D). These objects were placed in the corners of the arena $10 \mathrm{~cm}$ from the walls. Each mouse was placed in the center of the arena and allowed to explore the objects for $10 \mathrm{~min}$. During the delay period, all the objects were cleaned with alcohol to remove olfactory cues and any sawdust that had stuck to the object. In the test phase, two of the objects, e.g., B and D (which were both on the left or right of the arena), exchanged positions and the mouse was allowed to explore the objects for $10 \mathrm{~min}$. The time spent exploring the two objects that had changed position was calculated. The objects moved (i.e., those on the left or right) and the position of the objects in the sample phase were counterbalanced between mice.

\section{RNA isolation and quantitative real time-PCR (qPCR) analysis}


Total RNA was isolated from mouse hippocampus using RNeasy (Qiagen, Valencia, CA). The cDNA was synthesized using oligo-dT and random primers (TaKaRa, Dalian, China) for qPCR (ABI Prism 7900HT; Applied Biosystems, Foster City, CA) with commercial primers generated for the system. Relative expression was calculated using the $2-\triangle \Delta \mathrm{Cq}$ method. GAPDH was used as the internal control. Primers were listed in Table S1.

\section{Microarray}

Microarray was performed in hippocampus from 4-month-old F1 and F2 offspring. The raw microarray data GSE147039 is available at the NCBI Gene Expression Omnibus (GEO) database (http://www.ncbi.nlm.nih.gov/geo/). The probe set IDs were converted into the corresponding gene symbol using the annotation information derived from platform GPL6887. If multiple probesets correspond to one gene, the mean expression values of those probesets were obtained. The limma package V3.34.9 in R was used to identify the differentially expressed genes (DEGs) in F1 and F2 hippocampus compared with control group [21]. The DEGs were screened out according to adjusted Pvalue $<0.05$ and |log2FoldChange| $>1$. The clusterProfiler V3.8.0 in $\mathrm{R}$ was used to identify and visualize the Gene ontology (GO) terms and Kyoto Encyclopedia of Genes and Genomes (KEGG) pathways enriched by DEGs [22]. P-value < 0.05 was considered as a significant enrichment. GSEA of KEGG gene sets was run using 1,000 gene_set permutations by clusterProfiler. The normalized enrichment score (NES) was regarded as the primary statistic for examining GSEA enrichment results.

\section{Reduced representation bisulfite sequencing}

Reduced representation bisulfite sequencing (RRBS) was performed in sperm obtained from the caudal epididymis of 4-month-old F1 male mice (Genergy Biotechnology Co., Ltd., Shanghai, China). Briefly, $5 \mu \mathrm{g}$ genomic DNA was digested using the methylation-insensitive restriction enzyme Mspl (New England Biolabs, Beverly, MA, USA). A Qiagen Mini Purification kit (Qiagen, Hilden, Germany) was used to purify the digested products. Then, the ends of each restriction fragment were filled in and adenosine was added at the 3 '-end. Methylated paired-end Illumina adapters were ligated to the ends of the DNA fragments using T4 DNA ligase, and fragments sized 100-200 bp were purified by agarose gel extraction. The purified fragments were treated with sodium bisulfite and then amplified by PCR. The final PCR products were sequenced on HiSeq 2500 (Illumina Inc., San Diego, CA, USA). Differentially methylated loci (DML) and differentially methylated regions (DMRs) were analyzed based on a Bayesian approach [23], summarized as follows as our previous study [5]: two groups were modeled according to the Bayesian stratification model, and the Wald test was applied to each locus to get a $p$ value for each $\mathrm{CpG}$ site. For each $\mathrm{CpG}$ site, a difference in methylation value between two groups $\geq 5 \%$ and a posteriori probability of Wald test $\geq 0.95$ was considered to be a DML. A methylation region was defined as a DMR when it met these three criteria: (1) the length of this region was at least $50 \mathrm{bp}$; (2) the region contained no less than three $\mathrm{CpG}$ sites; (3) the proportion of DMLs in this region was no less than $50 \%$. When a DMR showed no less than $50 \%$ overlap with one element of the gene, it was defined as a differentially methylated gene (DMG). RRBS reads were mapped to reference mouse genome (mm10) by Bismark 
(version 0.16.3). DSS V2.30.1 in R was used to detect DMRs. The RRBS data reported in this paper have been deposited in the GEO database with accession number GSE142502.

\section{Statistical analysis}

Data are shown as the mean \pm SEM. For all behavioral studies, two-tailed unpaired Student's t-test was used to analyze the significance between groups. The t-test and Benjamini-Hochberg method were used to calculate the P-value and FDR of microarray, respectively. GO analysis was performed using a hypergeometric distribution test. The statistical test utilized by GSEA is the Kolmogorov-Smirnov statistical test. All the statistical analyses were conducted with GraphPad Prism 7 (GraphPad Software, Inc) and R (version 3.6.2). Differences were considered statistically significant at $p<0.05$.

\section{Results}

\section{Intrauterine hyperglycemia affects cognition of both F1 and F2 male offspring}

We introduced moderate hyperglycemia during pregnancy through injection of STZ. Male F1 adults were then intercrossed to unexposed females to obtain F2 offspring (Fig. 1a). Both F1 and F2 offspring were analyzed in behavioral tests at young adulthood at 3-4 months old.

The spontaneous explorative and locomotor activity was assessed in the open field test. F1-GDM mice showed normal total explorative distance as well as the explorative activity in the central area (Fig. 1b). The spatial working memory was examined using the Y-maze. F1-GDM mice performed worse than control in Y-maze (Fig. 1c), suggesting impaired spatial memory. The similar result was observed in novel object test (NOR). In the NOR test, the amount of time spent with the novel object compared with the total time spent exploring both object represents an index of recognition memory. Compared to F1-control, F1GDM mice spent less times investigating the novel object despite similar total exploration times, revealing a robust memory deficit (Fig. 1d).

Similar to F1 mice, F2-GDM mice showed normal locomotor activity (Fig. 1e), but displayed a significant deficit in spatial memory in the Y-maze test although there was no obvious difference in NOR test or object-in-place task (OiPT) associative recognition memory (Fig. 1f-h).

\section{Altered transcriptome profiling in adult hippocampus from F1 and F2-GDM}

The Gene expression patterns of hippocampus from 4-month-old F1 and F2 offspring ( $\mathrm{n}=5$ per group) were analyzed using microarray to investigate the effect of long-term reprogramming caused by intrauterine hyperglycemia. By using a stringent threshold and significant criteria of adjusted P-value < 0.05 and |log2FoldChange| $>1$, a total of 451 DEGs including 218 up-regulated and 234 down-regulated genes were identified in F1-GDM compared to Control, and, 1050 DEGs including 511 up-regulated and 
539 down-regulated genes were identified in F2-GDM compared to Control (Fig. 2a, 2c, Table S2, Table S3).

Enrichment curves computed by Gene set enrichment analysis (GSEA) of F1 hippocampi were highly consistent with F2. GSEA of the hippocampi transcriptome in F1 and F2-GDM vs. Control revealed the robust enrichment of curated gene sets for axon guidance and Wnt signaling pathway, indicating that the expression of member genes were decreased in the F1-GDM and F2-GDM group (NES < 0 ), although the changes of F2-GDM were not as significant as F1-GDM. GSEA also provided insights into changes of activated pathways in F1-GDM and F2-GDM, including cocaine addiction, dopaminergic synapse and neuroactive ligand-receptor interaction (NES > 0) (Fig. 2b, 2d, Table S4,Table S5).

Gene ontology (GO) analysis revealed that DEGs were mainly enriched in 'learning or memory', 'cognition', 'neuron to neuron synapse', 'postsynaptic specialization', and 'cAMP-mediated signaling'. There were several shared GO terms in both F1 and F2 hippocampus (Fig. 2e).

\section{The overlapped DEGs of hippocampus in both F1 and F2 offspring}

By overlapping DEGs of hippocampus in F1 and F2 offspring, we found that there were 106 genes upregulated in both F1-GDM and F2-GDM mice compared to control mice, and 117 genes down-regulated in both F1-GDM and F2-GDM mice. There is no differentially expressed gene showing inconsistent tendency in F1-GDM and F2-GDM mice (Fig. 3a). According to the significance of the difference and gene function, we verified some meaningful candidate genes by qPCR, including Akap7, Atp6ap2, Camk2b, Dlgap1, Drd1, Gpr88, Map1b, Penk, S100b, Tanc1, Tubb2b, Wnt5a, Zeb2 (Fig. 3b). The results of qPCR were consistent with that of the microarray. Enrichment analysis of 223 shared DEGs indicated that GO terms were mainly involved in biological process such as 'negative regulation of nervous system development', 'regulation of dopamine receptor signaling pathway', 'forebrain development', 'negative regulation of neuron differentiation', 'axon development', 'regulation of neurotransmitter transport and synapse organization' and so on (Fig. 3c,Table S6).

\section{The overlapped differentially methylated genes of F1-GDM sperm and differentially expressed genes of F2-GDM hippocampus}

The F1-GDM male were exposed to hyperglycemia in utero. Therefore the cognitive impairment could be due to direct disruption of neurodevelopment by hyperglycemia. In comparison, cognition impairment and gene expression chagnes in F2-GDM is most likely caused by epigenetic mechanisms such as DNA methylation because F2-GDM is from F1-GDM males breeding with unexposed naïve females. We therefore used genome methylation sequencing to search for differentially methylated loci of sperm between control and F1-GDM. Intrauterine hyperglycemia resulted in 408 DMLs associated with 345 genes that were then used in enrichment analysis (Table S7). The DMLs were distributed in the upstream $2 \mathrm{k}(8.58 \%), 5^{\prime}$-untranslated region (5'-UTR, 1.47\%), coding sequence (CDS, $23.28 \%$ ), introns (56.37\%), 3'UTR (5.15\%), downstream 2 k (5.15\%) (Fig. 4a). GO analysis identified a cluster of differentially methylated genes that were strongly related to 'neuron development', 'neuron differentiation' and 'organ 
growth' (Fig. 4b). By overlapping differentially methylated genes of sperm in F1 offspring and expressed genes of hippocampus in F2 offspring, we found 56 hypermethylated genes in F1-GDM sperm compared to Control. These 56 genes were down-regulated in F2-GDM hippocampus compared to Control (Fig. 4c). GO analysis showed that 56 genes were enriched in 'neuron to neuron synapse', 'postsynaptic density/specialization', 'neuron projection development', 'structural constituent of synapse/postsynapse', including Akap7, Camk2b, Dlgap1, Tanc1, Tubb2b, Wnt5a and Zeb2, most of which were down-regulated in hippocampus of both F1-GDM and F2-GDM (Fig. 2b). Additionally, within the 'nerve system development, GO:0007399' term in MGI database (http://www.informatics.jax.org/), a network of hypermethylated genes related to axon guidance ( $\mathrm{Htr7})$, positive regulation of astrocyte differentiation (Fryl), negative regulation of oligodendrocyte differentiation (Nfix), regulation of postsynaptic density assembly (Hoxb3), had a tendency for low expression in the F2-GDM versus the Control samples (Table 1). Of these genes, hypermethylated CpGs was most frequent in CDS regions, followed closely by intron regions. 
Table 1

The crucial genes associated with central nervous system development are hypermethylated in F1-GDM sperm and down-regulated expression in F2-GDM hippocampus.

\section{RRBS of Sperm}

(F1-GDM vs. Ctrl)

\begin{tabular}{|c|c|c|c|c|c|c|c|}
\hline Symbol & Start & End & $\begin{array}{l}\text { meanMethy } \\
\text { Ctrl }\end{array}$ & $\begin{array}{l}\text { meanMethy } \\
\text { F1-GDM }\end{array}$ & Element & $\log \mathrm{FC}$ & $\begin{array}{l}\text { adj. } P \\
\text { Value }\end{array}$ \\
\hline Tubb2b & 34127486 & 34127641 & $16.03 \%$ & $81.88 \%$ & CDS & -3.0865 & 0.001 \\
\hline $\mathrm{Htr} 7$ & 35969594 & 35969756 & $7.22 \%$ & $73.70 \%$ & $\begin{array}{l}\text { CDS 3'- } \\
\text { UTR }\end{array}$ & -1.7638 & 0.249 \\
\hline Dlgap1 & 70516618 & 70516771 & $6.80 \%$ & $86.87 \%$ & CDS & -1.2927 & 0.186 \\
\hline Tanc1 & 59843298 & 59843455 & $65.81 \%$ & $81.41 \%$ & CDS & -1.1135 & 0.026 \\
\hline Zeb2 & 44988693 & 44988893 & $14.49 \%$ & $85.39 \%$ & CDS & -0.7887 & 0.645 \\
\hline Wnt5a & 28518390 & 28522909 & $26.62 \%$ & $76.36 \%$ & $\begin{array}{l}\text { CDS } \\
\text { Intron }\end{array}$ & -0.5713 & 0.211 \\
\hline Hoxb3 & 96345925 & 96346054 & $7.08 \%$ & $28.65 \%$ & CDS & -0.4266 & 0.644 \\
\hline $\mathrm{Nfix}$ & 84784452 & 84784657 & $27.85 \%$ & $45.75 \%$ & Intron & -0.2088 & 0.678 \\
\hline $\mathrm{Nfix}$ & 84704134 & 84704384 & $38.83 \%$ & $64.20 \%$ & Intron & -0.2088 & 0.678 \\
\hline Akap7 & 25251588 & 25251727 & $25.82 \%$ & $77.69 \%$ & Intron & -0.2014 & 0.529 \\
\hline Camk2b & 6010025 & 6010230 & $49.49 \%$ & $89.20 \%$ & Intron & -0.1977 & 0.253 \\
\hline
\end{tabular}

\section{Discussion}

The association of GDM with offspring cognitive deficits has been investigated in a number of epidemiological studies [24, 25]. Further, a systematic review and meta-analysis found that according to 19 articles among 18,681 exposed and more than 2.8 million control participants, exposure to maternal pre-existing diabetes in pregnancy was not only related to an impaired intelligence ability in the offspring, but also increase the risk of autism spectrum disorders [26]. The data suggest there is a signal that GDM may associated with adverse neurocognitive and behavioral outcomes past the neonatal period. However, 
the underlying mechanisms leading to a higher susceptibility of the progeny to develop cognitive abnormalities later in life involve a complex pathophysiological change.

Maternal metabolic disorders could bring about sex-specific changes in the neurodevelopmental process of growing fetus. It is noteworthy that the male offspring are at a higher risk of developing neurodevelopmental disorders. Previous studies showed that fetal exposures to adverse maternal environment are significant risk factors for neuropsychiatric disease predisposition, in particular in male offspring [27-29]. In our previous study, we also found that the effect of intrauterine hyperglycemia on male offspring was more obvious than female, with parental characteristics and sex-specific transmission [4]. Therefore, in this study we focused on the male offspring.

In both F1-GDM and F2-GDM male offspring of mice, there was no difference with control in the open field test, suggesting that the change of learning and memory should not be confounded by the lack of locomotor activity. As the hippocampus-dependent memory test, the $\mathrm{Y}$-maze showed the spontaneous spatial recognition was significantly decreased in F1-GDM offspring. In the NOR test, F1-GDM mice spent significantly less times investigating the novel object and control, suggesting the ability to recognize new items significantly decreased. It's interesting that the impaired working memory function was more obvious in the F2-GDM offspring. Although there was no difference in novel object recognition or objectin-place test between F2-GDM and control. All these phenotypes indicated that the intrauterine hyperglycemia exposure could result in impairment of cognition and memory not only in F1 but also in F2 male offspring.

The clinical and basical evidence have suggested that the disturbances in intellectual and behavioral functioning observed in the children of diabetic women are accompanied by modification of hippocampus structure and function. Investigation of the mechanisms responsible for maternal diabetesrelated changes in the development of hippocampus is helping to prevent from impaired cognitive and memory functions in offspring [30]. In this study, the DEGs of F1-GDM and F2-GDM offspring were mainly enriched in 'learning or memory', 'cognition' and 'neuron to neuron synapse'. By overlapping DEGs of hippocampus in F1-GDM and F2-GDM offspring, totally we found the same 106 up-regulated genes and 117 down-regulated genes. It is interesting that there is no differentially expressed gene showing inconsistent tendency in F1-GDM and F2-GDM mice. The function of these DEGs included regulation of nervous system development, neuron differentiation and axon development.

Some important overlapped DEGs of F1-GDM and F2-GDM offspring were screened and verified in our study. Camk2, the calcium/calmodulin-dependent kinase type II, is holoenzyme of the forebrain predominantly, which consists of heteromeric complexes of the Camk2a and Camk2b isoforms, regulating hippocampal synaptic plasticity and learning [31,32]. Dopamine receptor Drd1 agonist could result in Camk2 activation, glutamate receptor exocytosis, synaptic reorganization, and expression of early markers of hippocampal synaptic plasticity [33]. Wnt5a regulates neuronal morphogenesis during embryonic development, and maintains dendritic architecture of pyramidal neurons in the adult hippocampus, through activating Wnt/JNK and Wnt/Camk2 signaling [34,35]. As a core protein involved 
in neurogenic Wnt signaling pathways, Atp6ap2 is critical for proliferating adult neural stem cells and differentiating neuroblasts, essential in early brain development, adult hippocampal neurogenesis and in cognitive functions. Lack of Atp6ap2 leads to cognitive impairment and neurodegeneration, and mutations of Atp6ap2 in humans are associated with intellectual disability [36, 37]. Post-synaptic density proteins (PSD) play a critical role in regulating the density and activity of glutamate receptors. As a scaffold protein localized at the PSD of glutamatergic neurons, Dlgap1 knockout leads to disruption of protein interactions in the PSD, and deficits in sociability[38]. And, TANC1 is a PSD-95-interacting synaptic protein that contains multiple domains for protein-protein interactions, important for dendritic spine maintenance and spatial memory [39]. The protein kinase A anchoring protein Akap7, a member of tubulin genes family Tubb2b, microtubule-associated protein Map1b and some other genes associated with hippocampal function were also changed in F1-GDM and F2-GDM offspring.

The explicit mechanism of maternal effect on offspring are still unclear. A few studies indicated that maternal overnutrition could induce cognitive deficits across several generations [40]. For F1 offspring, as a mediator of stress effects on neurodevelopmental reprogramming, the placenta may play an important role in the transmission of the maternal adverse environment and effects on the developing brain [41]. Dysregulation of imprinted genes is a plausible mechanism linking maternal stressors with fetal growth [42]. For mechanism of transmission to F2 offspring, based on our previous research that intrauterine exposure alone is sufficient to cause the epigenetic inheritance in $\mathrm{F} 2$ offspring $[4,5]$, we mainly investigated the methylation status of F1 sperm, finding 56 genes down-regulated in F2-GDM hippocampus hypermethylated in F1-GDM sperm. Our result confirmed that the epigenetic memory carried by DNA methylation pattern could be reprogrammed in F1 germcell during fetal development in uterus.

In conclusion, in this study, with mouse model, we firstly investigated the intergenerational effect of intrauterine hyperglycemia on the cognition and memory in offspring and the potential molecular mechanism. The results showed that intrauterine hyperglycemia exposure could result in impairment of cognition and memory in both F1 and F2 male offspring. The differentially expressed genes in both F1 and F2 hippocampi were mainly enriched in learning or memory, cognition, and other neuron function. Further research found the altered methylated modification of sperm in F1 adult caused by intrauterine hyperglycemia exposure. Therefore, the epigenetic alteration may play important role in the intergenerational transmission of GDM-induced abnormal neurodevelopment. It is essential that future studies focus on identifying potential mechanism of the maternal effect on epigenetic regulation in the fetus and even their germ cells.

\section{Declarations}

\section{Ethics approval and consent to participate}

All animal protocols were reviewed and approved by the Zhejiang University Animal Care and Use Committee. 


\section{Consent for publication}

Not applicable

\section{Availability of data and material}

We have provided a private GEO link that the reviewers can use to access this data:

To review GEO accession GSE147039:

Go to https://www.ncbi.nlm.nih.gov/geo/query/acc.cgi?acc=GSE147039

Enter token ebcbgskybxojlej into the box

To review GEO accession GSE142502:

Go to https://www.ncbi.nlm.nih.gov/geo/query/acc.cgi?acc=GSE142502

Enter token angjqeiarrotzyd into the box

\section{Competing interests}

The authors declare that they have no competing interests.

\section{Funding}

This work was supported by the National Key Research and Development Plan (2017YFC1001300, 2018YFC1004500), National Natural Science Foundation of China (81971458 and 31671222), the Municipal Human Resources Development Program for Outstanding Young Talents in Medical and Health Sciences in Shanghai (2017YQ047), CAMS Innovation Fund for Medical Sciences (2019-12M-5064), and the Ferring Institute of Reproductive Medicine, a strategic collaborative research program of Ferring Pharmaceuticals and Chinese Academy of Sciences (FIRMA180309). The research is also supported by the Innovative Research Team of High-level Local Universities in Shanghai, the Fundamental Research Funds for the Central Universities, and Shanghai Municipal Key Clinical Specialty, Shanghai, China.

\section{Authors' contributions}

GD conceived the study. GD and $X L$ designed research. $K Z, J R, C Z, C T, P L, X S$ and $G D$ performed research. $K Z$, JR, JZ and $G D$ analyzed the data. $K Z, J R, J Z, C Z, C T, P L, X S, J S, H H, X L$ and $G D$ interpreted the data. $\mathrm{GD}$ and $\mathrm{KZ}$ wrote the manuscript with the input from other authors.

\section{Acknowledgements}

Not applicable

\section{References}


1. Barker DJ, Eriksson JG, Forsen T, Osmond C. Fetal origins of adult disease: strength of effects and biological basis. Int J Epidemiol. 2002;31(6):1235-9.

2. Radford EJ, Ito M, Shi H, Corish JA, Yamazawa K, Isganaitis E, Seisenberger S, Hore TA, Reik W, Erkek $S$, et al. In utero effects. In utero undernourishment perturbs the adult sperm methylome and intergenerational metabolism. Science. 2014;345(6198):1255903.

3. Chandna AR, Kuhlmann N, Bryce CA, Greba Q, Campanucci VA, Howland JG. Chronic maternal hyperglycemia induced during mid-pregnancy in rats increases RAGE expression, augments hippocampal excitability, and alters behavior of the offspring. Neuroscience. 2015;303:241-60.

4. Ding GL, Wang FF, Shu J, Tian S, Jiang Y, Zhang D, Wang N, Luo Q, Zhang Y, Jin F, et al. Transgenerational glucose intolerance with Igf2/H19 epigenetic alterations in mouse islet induced by intrauterine hyperglycemia. Diabetes. 2012;61(5):1133-42.

5. Ren J, Cheng Y, Ming ZH, Dong XY, Zhou YZ, Ding GL, Pang HY, Rahman TU, Akbar R, Huang HF, et al. Intrauterine hyperglycemia exposure results in intergenerational inheritance via DNA methylation reprogramming on F1 PGCs. Epigenetics Chromatin. 2018;11(1):20.

6. Bytoft B, Knorr S, Vlachova Z, Jensen RB, Mathiesen ER, Beck-Nielsen H, Gravholt CH, Jensen DM, Clausen TD, Mortensen EL, et al: Long-term Cognitive Implications of Intrauterine Hyperglycemia in Adolescent Offspring of Women With Type 1 Diabetes (the EPICOM Study). Diabetes Care 2016, 39(8):1356-1363.

7. Nielsen GL, Dethlefsen C, Sorensen HT, Pedersen JF, Molsted-Pedersen L. Cognitive function and army rejection rate in young adult male offspring of women with diabetes: a Danish populationbased cohort study. Diabetes Care. 2007;30(11):2827-31.

8. Dahlquist G, Kallen B. School marks for Swedish children whose mothers had diabetes during pregnancy: a population-based study. Diabetologia. 2007;50(9):1826-31.

9. Rizzo T, Metzger BE, Burns WJ, Burns K. Correlations between antepartum maternal metabolism and intelligence of offspring. N Engl J Med. 1991;325(13):911-6.

10. Veena SR, Krishnaveni GV, Srinivasan K, Kurpad AV, Muthayya S, Hill JC, Kiran KN, Fall CH. Childhood cognitive ability: relationship to gestational diabetes mellitus in India. Diabetologia. 2010;53(10):2134-8.

11. Fraser A, Almqvist C, Larsson H, Langstrom N, Lawlor DA. Maternal diabetes in pregnancy and offspring cognitive ability: sibling study with 723,775 men from 579,857 families. Diabetologia. 2014;57(1):102-9.

12. Vuong B, Odero G, Rozbacher S, Stevenson M, Kereliuk SM, Pereira TJ, Dolinsky VW, Kauppinen TM. Exposure to gestational diabetes mellitus induces neuroinflammation, derangement of hippocampal neurons, and cognitive changes in rat offspring. J Neuroinflammation. 2017;14(1):80.

13. He A, Zhang Y, Yang Y, Li L, Feng X, Wei B, Zhu D, Liu Y, Wu L, Zhang L, et al. Prenatal high sucrose intake affected learning and memory of aged rat offspring with abnormal oxidative stress and NMDARs/Wnt signaling in the hippocampus. Brain Res. 2017;1669:114-21. 
14. Kruse MS, Vega MC, Rey M, Coirini H. Sex differences in LXR expression in normal offspring and in rats born to diabetic dams. J Endocrinol. 2014;222(1):53-60.

15. Hami J, Sadr-Nabavi A, Sankian M, Balali-Mood M, Haghir H. The effects of maternal diabetes on expression of insulin-like growth factor-1 and insulin receptors in male developing rat hippocampus. Brain Struct Funct. 2013;218(1):73-84.

16. Kuang H, Sun M, Lv J, Li J, Wu C, Chen N, Bo L, Wei X, Gu X, Liu Z, et al. Hippocampal apoptosis involved in learning deficits in the offspring exposed to maternal high sucrose diets. J Nutr Biochem. 2014;25(9):985-90.

17. Haghir H, Hami J, Lotfi N, Peyvandi M, Ghasemi S, Hosseini M. Expression of apoptosis-regulatory genes in the hippocampus of rat neonates born to mothers with diabetes. Metab Brain Dis. 2017;32(2):617-28.

18. Vafaei-Nezhad S, Hami J, Sadeghi A, Ghaemi K, Hosseini M, Abedini MR, Haghir H. The impacts of diabetes in pregnancy on hippocampal synaptogenesis in rat neonates. Neuroscience. 2016;318:122-33.

19. Hami J, Vafaei-Nezhad S, Ivar G, Sadeghi A, Ghaemi K, Mostafavizadeh M, Hosseini M. Altered expression and localization of synaptophysin in developing cerebellar cortex of neonatal rats due to maternal diabetes mellitus. Metab Brain Dis. 2016;31(6):1369-80.

20. Golic M, Stojanovska V, Bendix I, Wehner A, Herse F, Haase N, Kraker K, Fischer C, Alenina N, Bader M, et al. Diabetes Mellitus in Pregnancy Leads to Growth Restriction and Epigenetic Modification of the Srebf2 Gene in Rat Fetuses. Hypertension. 2018;71(5):911-20.

21. Ritchie ME, Phipson B, Wu D, Hu Y, Law CW, Shi W, Smyth GK. limma powers differential expression analyses for RNA-sequencing and microarray studies. Nucleic Acids Res. 2015;43(7):e47.

22. Yu G, Wang LG, Han Y, He QY. clusterProfiler: an R package for comparing biological themes among gene clusters. Omics. 2012;16(5):284-7.

23. Feng $\mathrm{H}$, Conneely $\mathrm{KN}$, Wu H. A Bayesian hierarchical model to detect differentially methylated loci from single nucleotide resolution sequencing data. Nucleic Acids Res. 2014;42(8):e69.

24. Adane AA, Mishra GD, Tooth LR. Diabetes in Pregnancy and Childhood Cognitive Development: A Systematic Review. Pediatrics 2016, 137(5).

25. Fraser A, Lawlor DA. Long-term health outcomes in offspring born to women with diabetes in pregnancy. Curr Diab Rep. 2014;14(5):489.

26. Yamamoto JM, Benham JL, Dewey D, Sanchez JJ, Murphy HR, Feig DS, Donovan LE. Neurocognitive and behavioural outcomes in offspring exposed to maternal pre-existing diabetes: a systematic review and meta-analysis. Diabetologia. 2019;62(9):1561-74.

27. Braithwaite EC, Pickles A, Sharp H, Glover V, O'Donnell KJ, Tibu F, Hill J. Maternal prenatal cortisol predicts infant negative emotionality in a sex-dependent manner. Physiol Behav. 2017;175:31-6.

28. Lemaire V, Koehl M, Le Moal M, Abrous DN. Prenatal stress produces learning deficits associated with an inhibition of neurogenesis in the hippocampus. Proc Natl Acad Sci U S A. 2000;97(20):11032-7. 
29. Misra P, Ganesh S. Sex-biased transgenerational effect of maternal stress on neurodevelopment and cognitive functions. J Genet. 2018;97(2):581-3.

30. Hami J, Shojae F, Vafaee-Nezhad S, Lotfi N, Kheradmand H, Haghir H. Some of the experimental and clinical aspects of the effects of the maternal diabetes on developing hippocampus. World $\mathrm{J}$ Diabetes. 2015;6(3):412-22.

31. Borgesius NZ, van Woerden GM, Buitendijk GH, Keijzer N, Jaarsma D, Hoogenraad CC, Elgersma Y. betaCaMKII plays a nonenzymatic role in hippocampal synaptic plasticity and learning by targeting alphaCaMKII to synapses. J Neurosci. 2011;31(28):10141-8.

32. Kool MJ, Proietti Onori M, Borgesius NZ, van de Bree JE, Elgersma-Hooisma M, Nio E, Bezstarosti K, Buitendijk GHS, Aghadavoud Jolfaei M, Demmers JAA, et al. CAMK2-Dependent Signaling in Neurons Is Essential for Survival. J Neurosci. 2019;39(28):5424-39.

33. Kern A, Mavrikaki M, Ullrich C, Albarran-Zeckler R, Brantley AF, Smith RG. Hippocampal Dopamine/DRD1 Signaling Dependent on the Ghrelin Receptor. Cell. 2015;163(5):1176-90.

34. Arredondo SB, Guerrero FG, Herrera-Soto A, Jensen-Flores J, Bustamante DB, Onate-Ponce A, Henny P, Varas-Godoy M, Inestrosa NC, Varela-Nallar L. Wnt5a promotes differentiation and development of adult-born neurons in the hippocampus by noncanonical Wnt signaling. Stem Cells. 2020;38(3):42236.

35. Chen CM, Orefice LL, Chiu SL, LeGates TA, Hattar S, Huganir RL, Zhao H, Xu B, Kuruvilla R. Wnt5a is essential for hippocampal dendritic maintenance and spatial learning and memory in adult mice. Proc Natl Acad Sci U S A. 2017;114(4):E619-28.

36. Schafer ST, Han J, Pena M, von B Und Halbach, Peters O, Gage J. FH: The Wnt adaptor protein ATP6AP2 regulates multiple stages of adult hippocampal neurogenesis. $J$ Neurosci. 2015;35(12):4983-98.

37. Bracke A, Schafer S, von Bohlen Und Halbach V, Klempin F, Bente K, Bracke K, Staar D, van den Brandt J, Harzsch S, Bader M, et al. ATP6AP2 over-expression causes morphological alterations in the hippocampus and in hippocampus-related behaviour. Brain Struct Funct. 2018;223(5):2287-302.

38. Coba MP, Ramaker MJ, Ho EV, Thompson SL, Komiyama NH, Grant SGN, Knowles JA, Dulawa SC. Dlgap1 knockout mice exhibit alterations of the postsynaptic density and selective reductions in sociability. Sci Rep. 2018;8(1):2281.

39. Han S, Nam J, Li Y, Kim S, Cho SH, Cho YS, Choi SY, Choi J, Han K, Kim Y, et al. Regulation of dendritic spines, spatial memory, and embryonic development by the TANC family of PSD-95interacting proteins. J Neurosci. 2010;30(45):15102-12.

40. Sarker G, Peleg-Raibstein D. Maternal Overnutrition Induces Long-Term Cognitive Deficits across Several Generations. Nutrients 2018, 11(1).

41. Bronson SL, Bale TL. The Placenta as a Mediator of Stress Effects on Neurodevelopmental Reprogramming. Neuropsychopharmacology. 2016;41(1):207-18.

42. Argyraki M, Damdimopoulou P, Chatzimeletiou K, Grimbizis GF, Tarlatzis BC, Syrrou M, Lambropoulos A. In-utero stress and mode of conception: impact on regulation of imprinted genes, fetal 
development and future health. Hum Reprod Update. 2019;25(6):777-801.

\section{Electronic Appendix}

Table S1 The primers for qPCR. Sequences are shown in the $5^{\prime}$ to 3 'direction.

Table S2 Differentially expressed genes in hippocampus of F1-GDM male offspring.

Table S3 Differentially expressed genes in hippocampus of F2-GDM male offspring.

Table S4 GSEA-based KEGG-enrichment analysis of gene sets of F1-GDM

Table S5 GSEA-based KEGG-enrichment analysis of gene sets of F2-GDM

Table S6 GO analysis of overlapped DEGs of F1 and F2 offspring.

Table S7 Differentially methylated genes in sperm of F1-GDM mice.

\section{Figures}


a

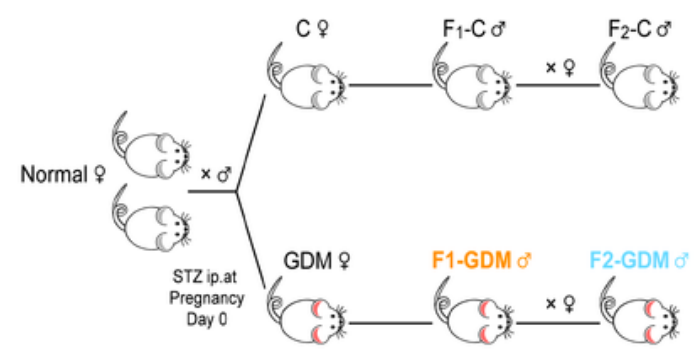

C

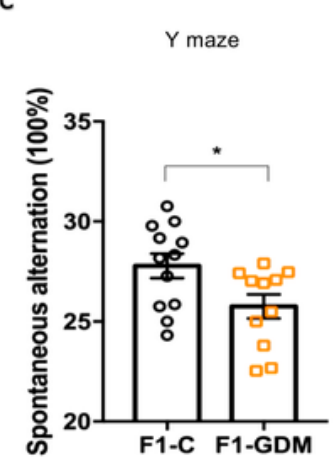

f

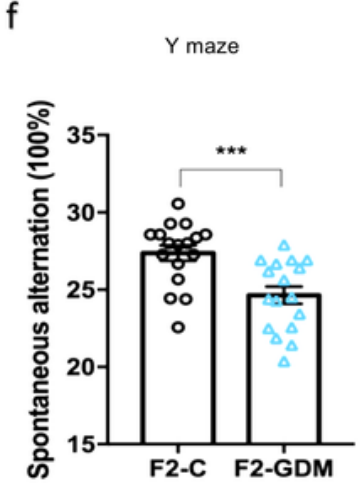

b

Open field test

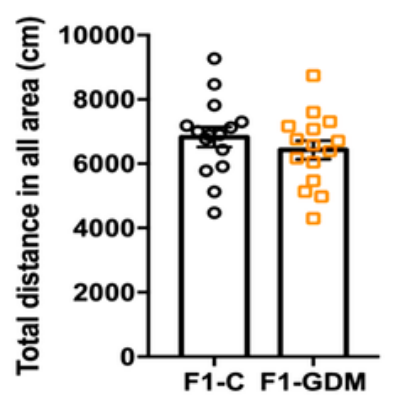

Open field test
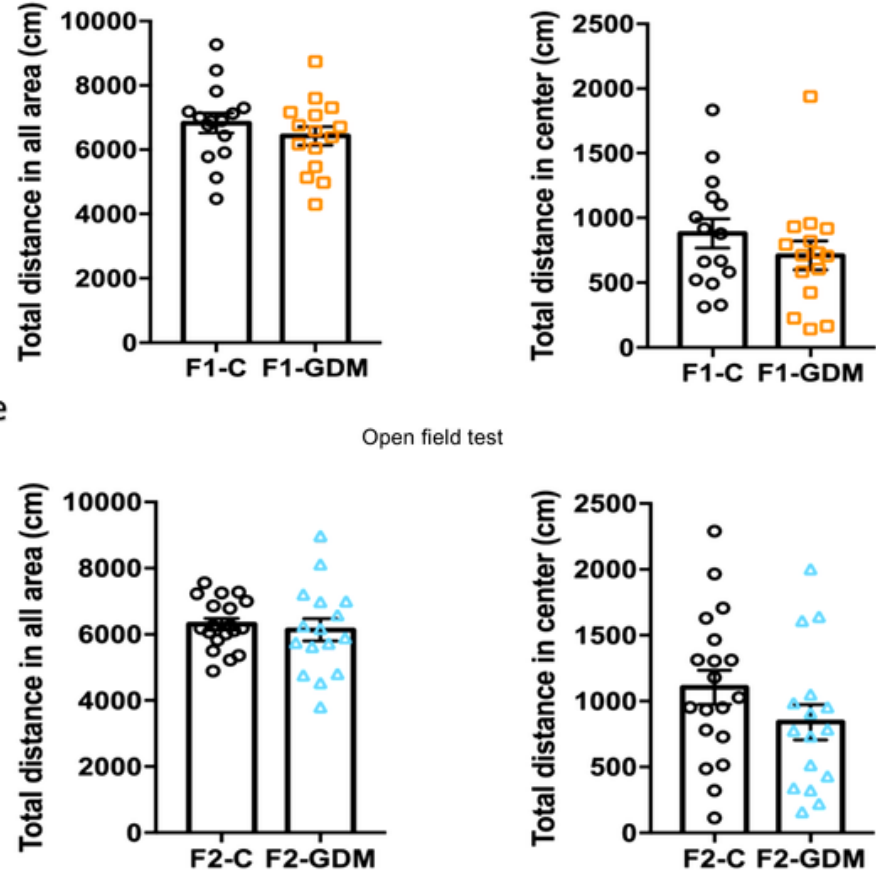

h

d

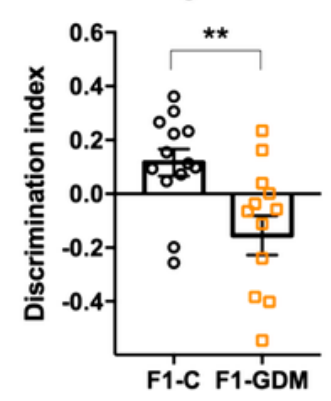

g

Novel object recognition

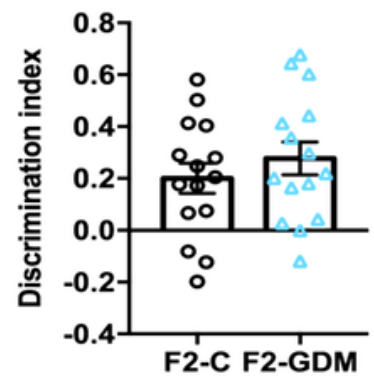

$\mathrm{h} \quad$ Object in place test

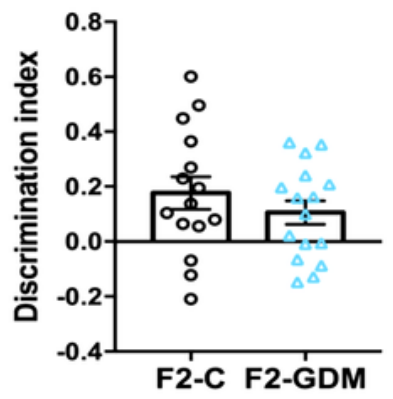

\section{Figure 1}

Experimental design and behavioral tests of F1 and F2 offspring. (a) Experimental design. (b) Open field test of F1 offspring of control (F1-C) and GDM (F1-GDM) mice (nF1-C =15, nF1-GDM=15 male 3-monthold mice). (c) Y maze test of F1-C and F1-GDM mice ( $\mathrm{nF1-C}=12$, nF1-GDM=11 male 3-month-old mice). (d) Novel object recognition test of F1-C and F1-GDM mice ( $\mathrm{nF1-C}=13, \mathrm{nF1-GDM=13}$ male 3-month-old mice). (e) Open field test of F2 offspring of control (F2-C) and GDM (F2-GDM) mice (nF2-C =19, nF2- 
$\mathrm{GDM}=16$ male 3-month-old mice). (f) $\mathrm{Y}$ maze test of F2-C and F2-GDM mice ( $\mathrm{nF2}-\mathrm{C}=17, \mathrm{nF2}-\mathrm{GDM}=17$ male 3-month-old mice). (g) Novel object recognition test of F2-C and F2-GDM mice ( $\mathrm{nF2}-\mathrm{C}=15$, $\mathrm{nF2}$ $\mathrm{GDM}=15$ male 3-month-old mice). (h) Object in place test of F2-C and F2-GDM mice (nF2-C =15, nF2$\mathrm{GDM}=15$ male 3-month-old mice). Data were analyzed by two-tailed unpaired t test. ${ }^{\mathrm{P}}<0.05$ vs. F1-C; **P $<0.01$ vs. F1-C; ***P $<0.001$ vs. F2-C.

a

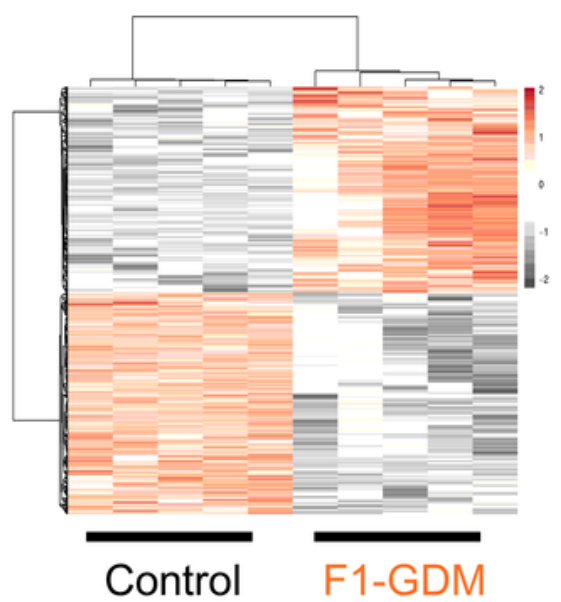

C

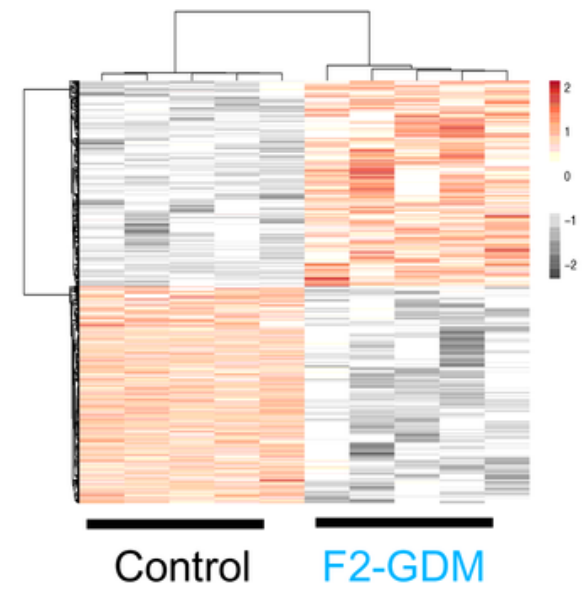

b
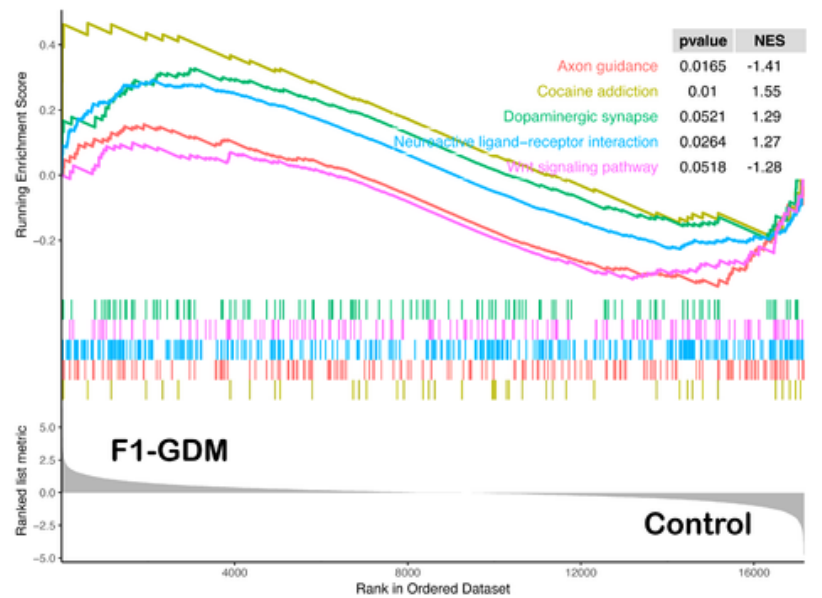

d

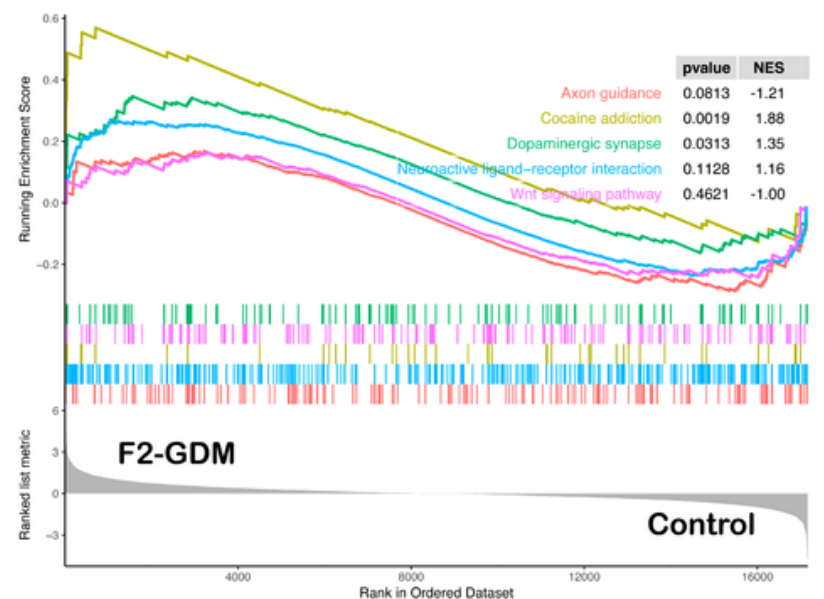

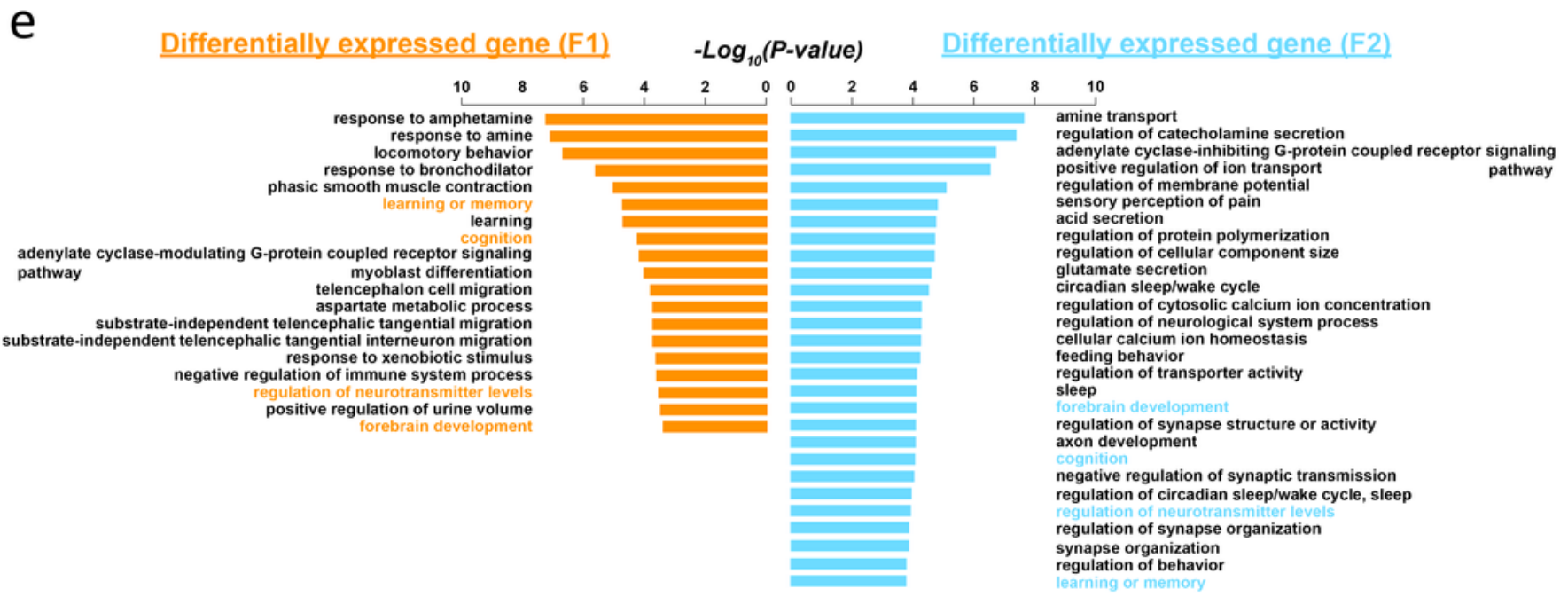

Figure 2 
Altered transcriptome profiling in adult hippocampus from F1 and F2-GDM. (a, c) Heat map of differentially expressed genes (DEGs) between control and F1-GDM mice, control and F2-GDM mice. (ncontrol =5, nF1-GDM=5, nF2-GDM=5 male 4-month-old mice). (b, d) GSEA of microarray data identified five enriched gene sets in F1 and F2 offspring. (e) GO analysis of DEGs in F1 and F2 offspring. NES, absolute value of normalized enrichment score.

a

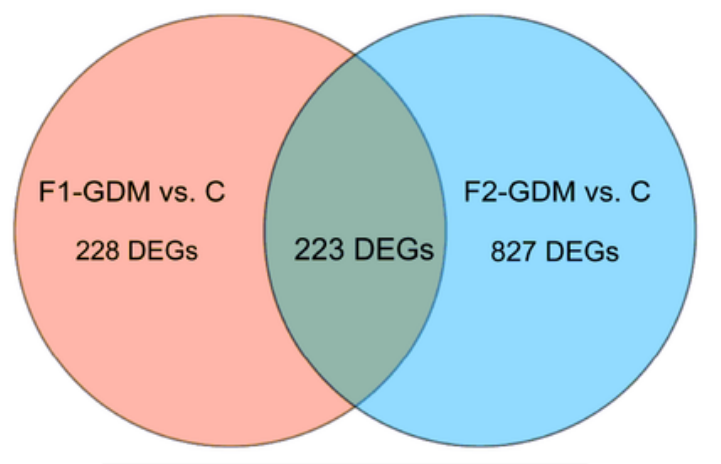

Shared DEGs, across 223 genes

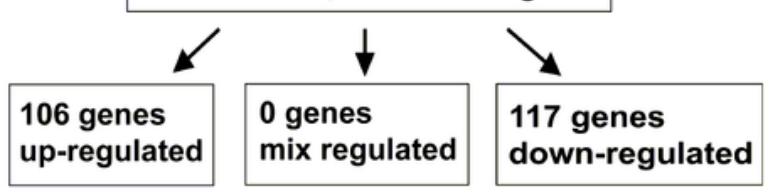

C

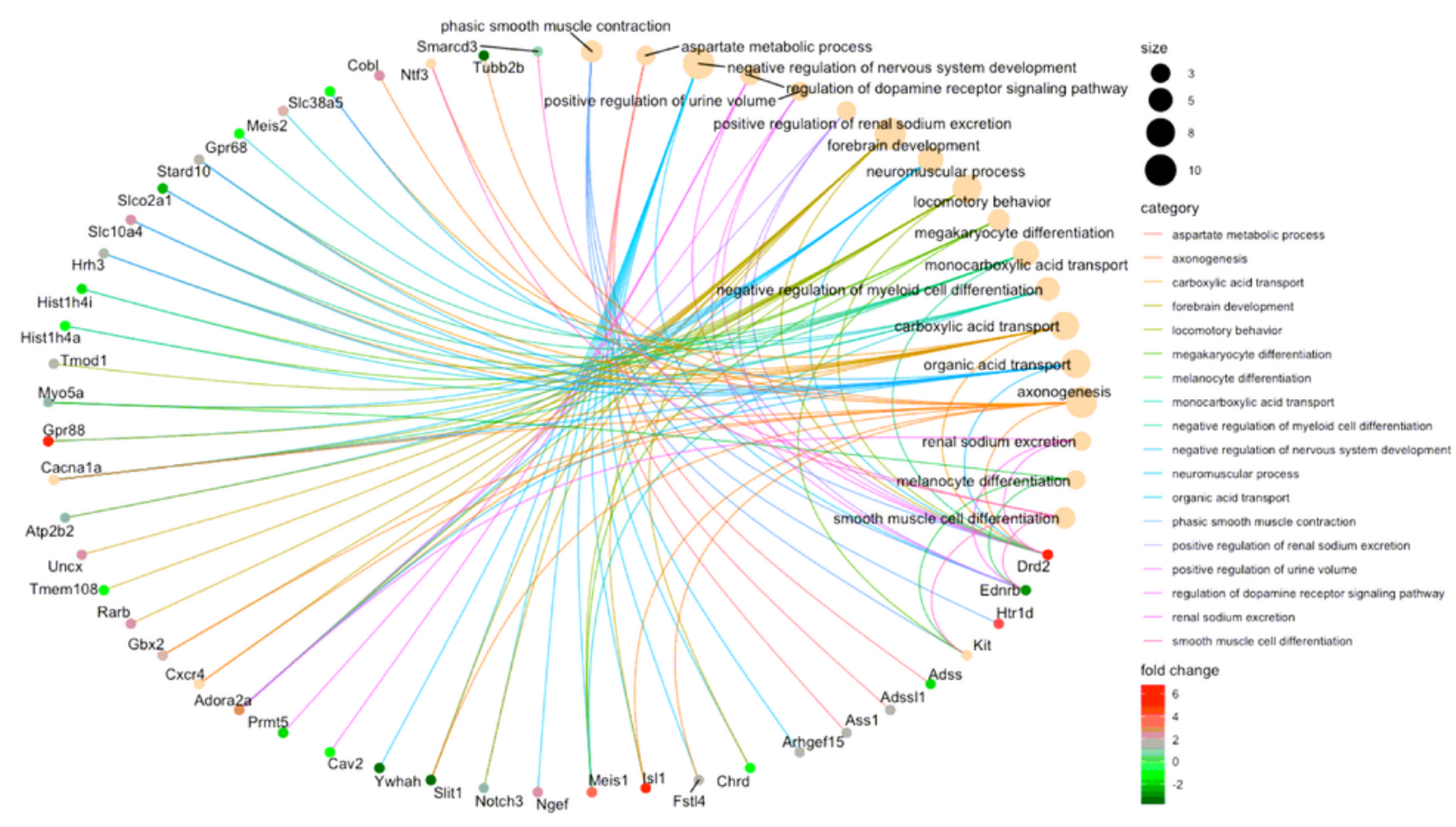

b

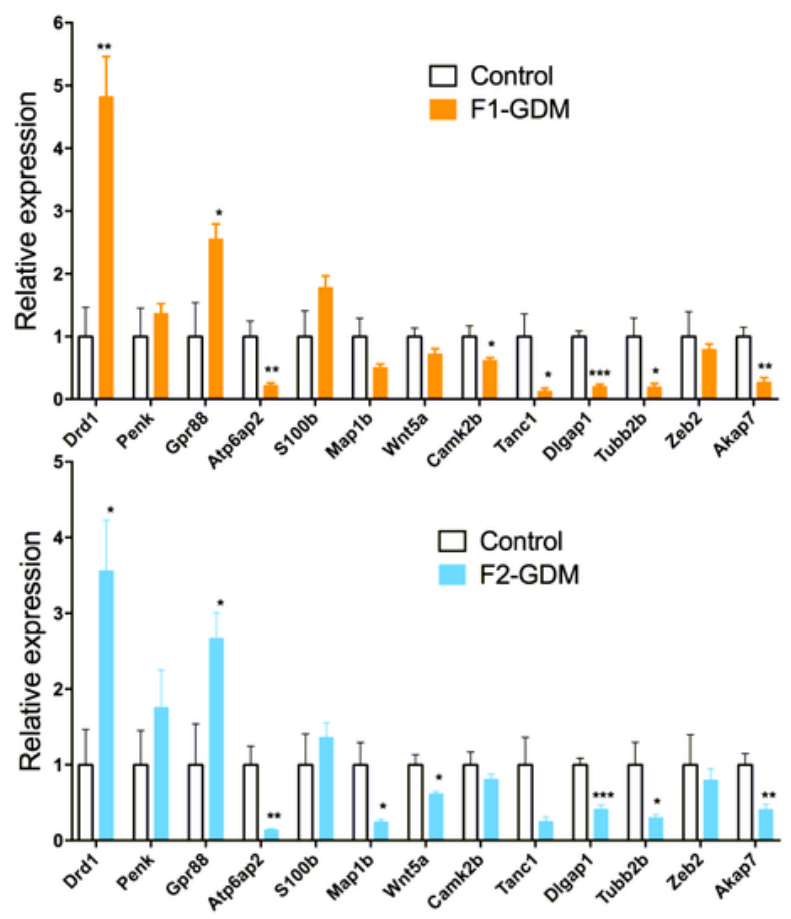

Figure 3 
The overlapped differentially expressed genes of hippocampus in both F1-GDM and F2-GDM offspring (a) Venn diagram of differential genes overlapped between F1-GDM vs. control and F2-GDM vs. control. (b) The expression of meaningful shared DEGs in F1 and F2 offspring (ncontrol =5, nF1-GDM=5, nF2$\mathrm{GDM}=5$ male 4-month-old mice). (c) Enrichment analysis of shared DEGs in F1 and F2 offspring. Data were analyzed by two-tailed unpaired t test. ${ }^{*} \mathrm{P}<0.05$ vs. control; $* * P<0.01$ vs. control; $* \star * P<0.001$ vs. control.

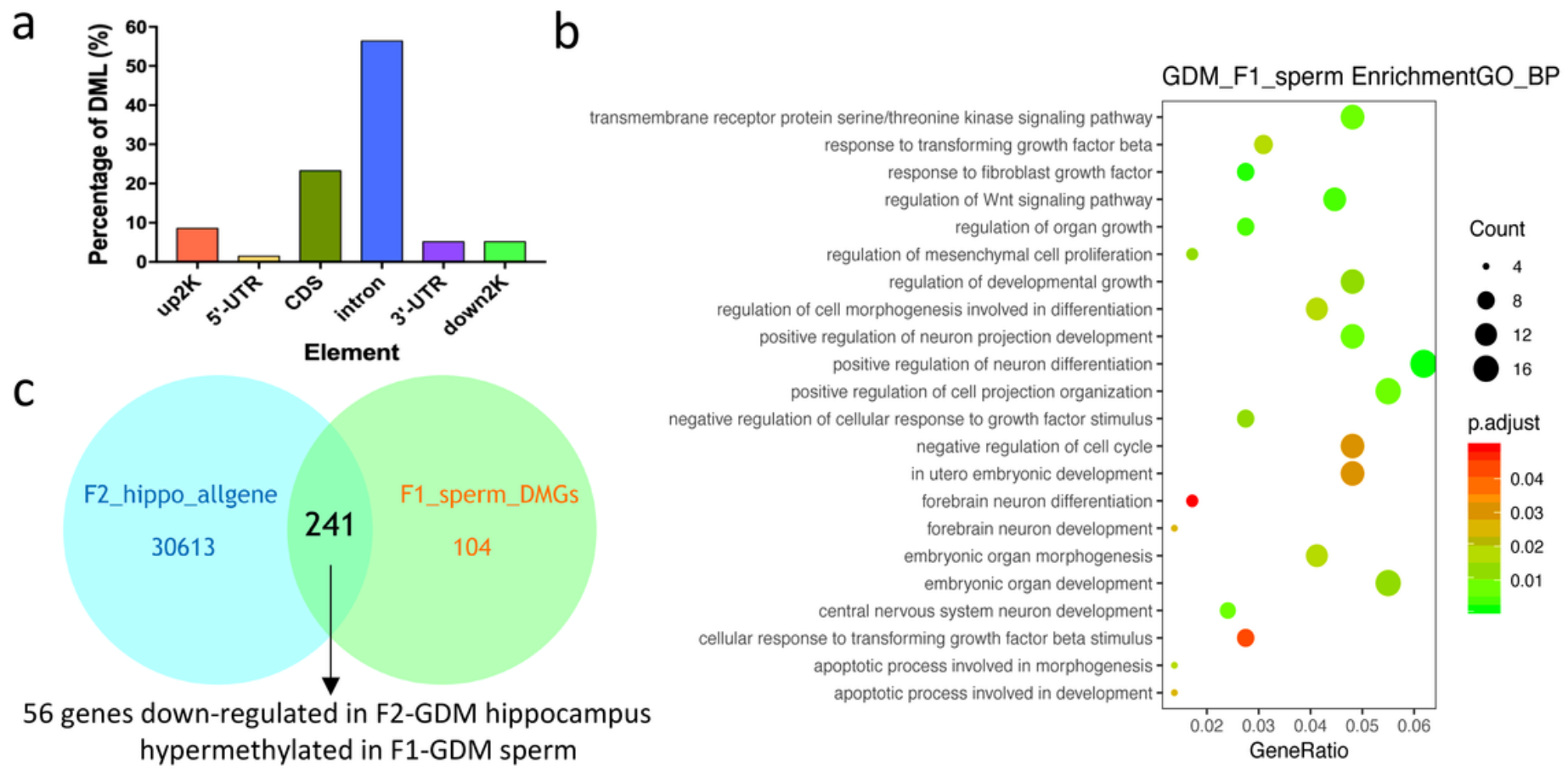

\section{Figure 4}

The overlapped differentially methylated genes of F1-GDM sperm and differential expressed genes of F2GDM hippocampus (a) Distribution of differentially methylated peaks within the genome in F1-GDM sperm. (b) GO analysis of differentially methylated genes in F1-GDM sperm. (c) Venn diagram of differentially methylated genes of F1-GDM sperm overlapped all genes of F2-GDM hippocampus.

\section{Supplementary Files}

This is a list of supplementary files associated with this preprint. Click to download.

- TableS7.xlsx

- TableS6.xIsx

- TableS5.xIsx

- TableS4.xlsx

- TableS3.xIsx

- TableS2.xIsx 
- Tables1.xlsx

Page 22/22 